\title{
Genomic characteristics of adhesion molecules in patients with symptomatic pulmonary embolism
}

\author{
YUAN XIE*, QIANGLIN DUAN*, LEMING WANG, ZHU GONG, \\ QIANG WANG, HAOMING SONG and HAO WANG
}

Department of Cardiology, Tongji Hospital, School of Medicine, Tongji University, Shanghai, P.R. China

Received April 13, 2012; Accepted June 1, 2012

DOI: $10.3892 / \mathrm{mmr} .2012 .940$

\begin{abstract}
The aim of this study was to identify the differences in the expression of cell adhesion molecule-related mRNAs between symptomatic pulmonary embolism (PE) and a control group, and to investigate the interactions among activated leukocytes, platelets and endothelial cells. Whole human gene chip was applied to detect the expression of cell adhesion molecule-related mRNAs in symptomatic PE and in the control group, and statistical analysis was performed. In patients with $\mathrm{PE}$, the expression of the majority of integrin mRNAs located on leukocytes and platelets was significantly upregulated. The expression of mRNAs related to L-selectin and P-selectin glycoprotein ligand was significantly upregulated, while the expression of mRNA related to E-selectin was significantly downregulated. The expression of mRNAs related to classic cadherins and protocadherins was downregulated, and the expression of mRNAs related to vascular endothelial cadherin was significantly downregulated; the expression of mRNAs related to the immunoglobulin superfamily had no obvious difference between the 2 groups. In conclusion, we demonstrated that, in symptomatic PE patients, the adhesion of leukocytes and platelets was enhanced; the activation of endothelial cells was obviously weakened; the adherens junctions among endothelial cells were weakened, with the endothelium becoming more permeable.
\end{abstract}

\section{Introduction}

Pulmonary embolism (PE) consists of acute pulmonary thromboembolism (APE) and chronic thromboembolic pulmonary hypertension (CTEPH). PE together with deep venous thrombosis (DVT) is termed venous thromboembolism (VTE). PE

Correspondence to: Dr Leming Wang, Department of Cardiology, Tongji Hospital, School of Medicine, Tongji University, 389 Xincun Rd, Shanghai 200065, P.R. China

E-mail: wanglemin@tongji.edu.cn

*Contributed equally

Key words: symptomatic pulmonary embolism, cell adhesion molecules, mRNA has become an international health care problem due to its high morbidity, misdiagnosis rate and mortality (1)

More than a century ago, Vichrow proposed a theory known as Virchow's triad, stating that thrombosis results from a combination of hemodynamic changes (stasis, turbulence), hypercoagulability and vessel wall injury (2). This theory forms the basis of the pathogenesis of artery thrombosis and VTE. The American Collage of Chest Physicians (ACCP) proposed a risk stratification for VTE and recommended different methods of prevention for patients at different risk levels (3). As a matter of fact, some patients at the same risk level and in a similar external environment, develop VTE after surgery, but the majority do not. It is still not clear as to why the incidence of VTE increases with age and why patients with a malignant disease are prone to VTE. It is also still not clear as to why artery thrombus is a 'white clot' and VTE a 'red clot'. Although some patients with DVT/PE received warfarin continuously, and the D-dimer level was regulated within a good range, their pulmonary artery pressure still increased gradually and they eventually developed CTEPH. ACCP summarized the risk factors for VTE as: trauma, surgery, increasing age, malignancy, pregnancy, heart failure, immobility and estrogen-containing oral contraception $(3,4)$. Although guidelines for the prevention, diagnosis and treatment of VTE have been published 8 times from 1995 to 2008 and have been updated continuously, the clinical confusion associated with VTE remains. In 2008, Shackfored reviewed the records of 37,615 patients with symptomatic VTE on surgery services over the 10-year period since the initial publication of the ACCP guidelines, of which $84 \%$ were either in partial or complete compliance with the guidelines. The incidence of VTE, however, increased gradually over the years of the study, instead of decreasing (5). The possible reason for theory and clinical practice being separated is that the etiology and pathogenesis of VTE are not yet clear.

In 2006, Smeeth reported that VTE was associated with infection, especially in the first 2 weeks following infection (6). In 2010 it was also reported that VTE was found in multiple organs in a patient, who died of severe acute respiratory syndrome (SARS), indicating that a viral infection caused systemic VTE (7). In a previous study, we identified a viruslike structure in T cells in a patient with CTEPH, which proved that $\mathrm{T}$ cells were infiltrated by a virus (8). In 2011, it was also reported that $\mathrm{CD}^{+}$and $\mathrm{CD}^{+} \mathrm{T}$ cell-mediated immune deficiency or compromise occurred in patients with CTEPH (9) 
and acute PE (10). Our research focused on the correlation between VTE and infection, inflammation, and immunity.

There are numerous leukocytes aggregating at the site of a thrombus in VTE. The adhesion among leukocytes, endothelial cells and platelets occurs throughout the process of VTE $(11,12)$. Cell adhesion molecules (CAMs) are a type of glycoprotein expressed on the cell surface mediating cellcell and cell-matrix interactions, which are the basis of cell adhesion. CAMs participate in a series of physiological and pathological processes, including signal transduction and activation, morphogenic movements, cellular migrations, cell growth and differentiation, as well as inflammation, thrombosis, wound healing and metastasis. Hundreds of CAMs have been identified in humans and are divided into 4 families: integrins, selectins and the immunoglobulin and cadherin superfamily. In this research, a whole human gene expression chip was applied to detect the differences of CAM-related mRNA expression in patients with $\mathrm{PE}$ and in a control group. The correlations among CAMs in activated leukocytes, platelets and endothelial cells during the process of PE were investigated.

\section{Materials and methods}

Patient information. The 20 patients enrolled in the PE group were those who were admitted in hospital during the year 2007, and included 11 males and 9 females, with an average age of $70 \pm 14$ years (44-89 years old). All patients were diagnosed with PE on the basis of at least 2 of the following criteria: i) selective pulmonary arteriography showing a filling defect or blockage; ii) pulmonary ventilation perfusion scanning exhibiting single or multiple blood flow perfusion defects with normal or abnormal ventilation and mismatched ratio of ventilation/ perfusion; iii) other clinical characteristics, including a typical manifestation of PE, arterial blood gas analysis, D-dimer test, ultrasound cardiogram (UCG) and chest computerized tomography $(\mathrm{CT})$ supported the diagnosis and excluded other cardiac and pulmonary disorders. Another 20 patients with ischemic heart disease admitted during the same period, without PE, DVT and other congenital bleeding and thrombosis diseases with comparative clinical presentation (11 males, 9 females; 44-91 years of age with a mean age of $72 \pm 14$ ) were enrolled in the control group. The study protocol was approved by the local ethics committee and an informed consent was obtained from all the patients in accordance with the declaration of Helsinki.

Total RNA isolation. A total of $5 \mathrm{ml}$ of peripheral blood samples anti-coagulated with EDTA were drawn from patients suspected of having PE and from those without PE, immediately after being admitted to the hospital. Mononuclear cells were obtained through density gradient centrifugation with Ficoll solution and the remaining red blood cells were destroyed by erythrocyte lysis buffer (Qiagen, Hilden, Germany). Total mononuclear cell RNA was extracted with TRIzol (Invitrogen, Carlsbad, USA) and purified with Qiagen RNeasy column (Qiagen), according to the manufacturer's instructions. The isolated total RNA was tested and quantified using a Nanodrop ND-1000 spectrophotometer (Nanodrop Technology, Cambrige, UK).

Gene expression clip. Agilent G4112A Whole Human Genome Oligo Microarrays were purchased from Agilent (USA). A microarray is composed of 44,290 spots including 41,675 genes or transcripts, 314 negative control spots, 1,924 positive control spots and 359 blank spots. The functions of more than $70 \%$ of the genes in the microarray are already known. All patients were subjected to clip analysis.

Target preparation and microarray hybridization. The RNA samples of patients with confirmed diagnosis of PE and controls were labeled using the indirect labeling method. Briefly, $1 \mu \mathrm{g}$ of total RNA was reverse transcribed. Second strand cDNA was then produced and purified followed by in vitro transcription (IVT) with T7 RNA Polymerase. During IVT, the modified nucleotide, 5-(3-aminoallyl)-UTP (aaUTP) was incorporated into the cDNA. Subsequently, the fluorescent Cy3 was chemically coupled with the aaUTP which contains a reactive primary amino group on the $\mathrm{C} 5$ position of uracil. The dye incorporation rate was assessed with a Nanodrop ND-1000 spectrophotometer and was found to be between 1.2-1.4 pmol $/ \mu 1$. Hybridization was carried out using the Agilent Oligonucleotide Microarray in situ Hybridization Plus kit (p/n 5,184-3,568), according to the manufacturer's instructions. Briefly, $750 \mathrm{ng}$ of Cy3-labeled sample cDNA was subjected to fragmentation $\left(30 \mathrm{~min}\right.$ at $60^{\circ} \mathrm{C}$ ) and then hybridization on 44K Human Whole-Genome 60-mer oligo-chips (G4112F, Agilent Technologies) in a rotary oven $\left(10 \mathrm{rpm}, 60^{\circ} \mathrm{C}, 17 \mathrm{~h}\right)$. Slides were disassembled and washed in solutions I and II, according to the manufacturer's instructions.

$R T-P C R$. Three differential genes in the microarray were selected and their expressions were confirmed by RT-PCR. Among the genes with differential expressions, 3 genes were randomly selected and these genes and the house keeping gene (GAPDH) were subjected to RT-PCR. The relative expression levels were indicated as the expression of the target genes normalized to the expression of GAPDH $\left(2^{-\Delta \Delta C t}\right)$. The melting curve and the $2^{-\Delta \Delta C t}$-method were used to compare the differences in the expressions between the control and the PE group. The results from RT-PCR were consistent with from the microarray analysis.

Statistical analysis. The Agilent Feature Extraction software was used to collect the original data from the microarray, followed by an analysis with a robust multichip average (RMA). The gene intensity data between the PE and control group were compared with a random variance model-corrected t-test. Differentially expressed genes were identified from whole genomes. A p-value $<0.05$ was considered to indicate a statistically significant difference.

\section{Results}

\section{Integrin mRNA expression}

Leukocyte-related integrin mRNA expression. Among the 13 mRNAs, 7 were upregulated (of which 5 significantly) in the PE group, compared with the controls $(\mathrm{p}<0.05) ; 6$ were downregulated (of which 1 significantly) in the PE group $(\mathrm{p}<0.05)$ (Fig. 1A).

Platelet-related integrin $m R N A$ expression. Of the 7 mRNAs, ITGA2, ITGA5, ITGA6 and ITGB1 were also leukocyte-related integrin mRNAs. Five mRNAs were upregulated (of which 4 significantly) ( $\mathrm{p}<0.05) ; 2$ mRNAs were 
A

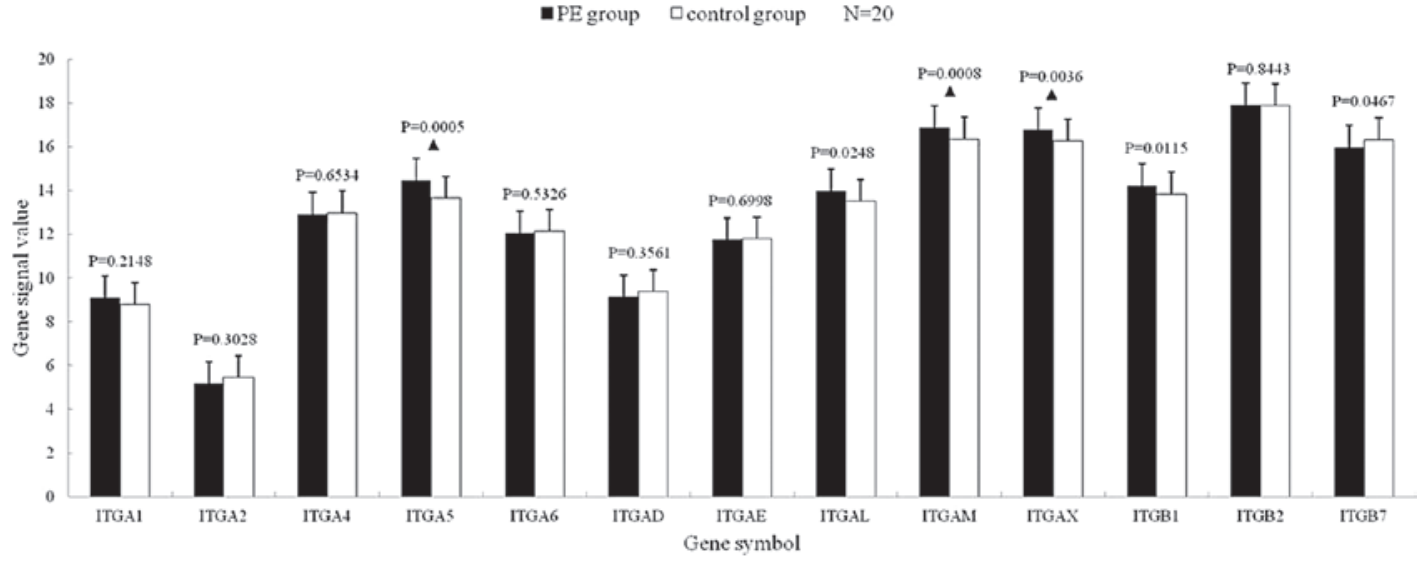

B

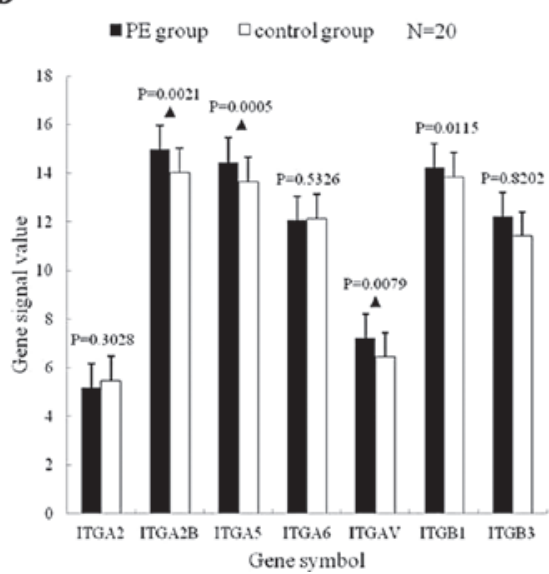

C

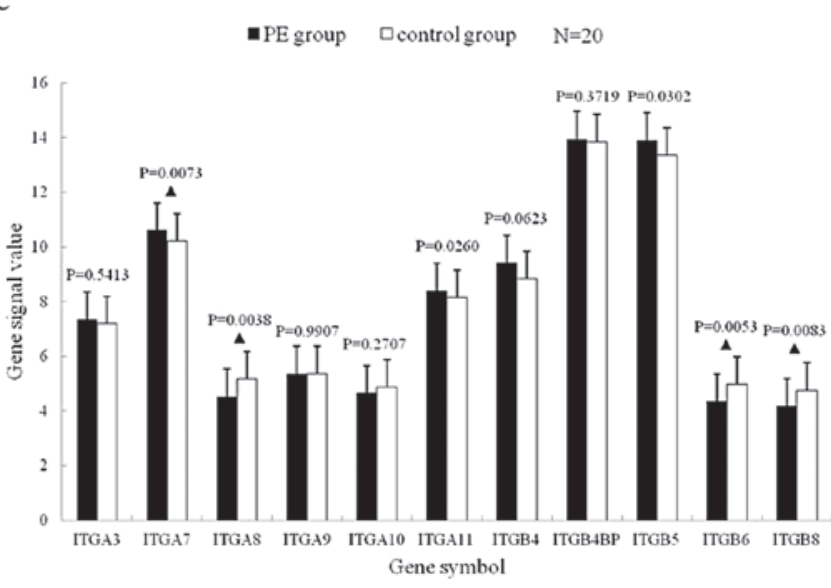

Figure 1. Expression of integrin mRNAs. (A) leukocyte-related integrin mRNA expression; (B) platelet-related integrin mRNA expression; (C) other integrin mRNA expression. ${ }^{*} \mathrm{p}<0.05 ;{ }^{\wedge} \mathrm{p}<0.01$

downregulated, but with no statistically significant difference ( $>0.05)$ (Fig. 1B).

Other integrin mRNA expression. Among the $11 \mathrm{mRNAs}$, 6 were upregulated (of which 3 significantly) in the PE group $(\mathrm{p}<0.05)$ and 5 were downregulated (of which 3 significantly) $(\mathrm{p}<0.01)$ (Fig. 1C).

Selectin mRNA expression. Among the 4 mRNAs, SELL and SELPLG mRNA expressions were significantly upregulated $(\mathrm{p}<0.01)$, while the SELE mRNA expression was significantly downregulated $(\mathrm{p}<0.01)$ (Fig. 2A).

Immunoglobulin superfamily mRNA expression. There were no statistically significant differences in the $5 \mathrm{mRNAs}$ between the PE and control group ( $\mathrm{p}>0.05)$ (Fig. 2B).

\section{Cadherin superfamily $m R N A$ expression}

Classic cadherin mRNA expression. Among the 13 mRNAs, 10 were downregulated (of which 7 significantly) in the PE group $(\mathrm{p}<0.05)$ and 3 were upregulated (of which 1 significantly) $(\mathrm{p}<0.05)$ (Fig. 2C).

Protocadherin mRNA expression. The 34 mRNAs consisted of 13 non-clustered and 21 clustered protocadherins. A total of 19 mRNAs were significantly downregulated $(\mathrm{p}<0.05)$, of which 9 more significantly $(\mathrm{p}<0.01) ; 3$ mRNAs were upregu- lated significantly $(\mathrm{p}<0.05)$, of which 2 more significantly $(\mathrm{p}<0.01)$ (Fig. 3).

Flamingo cadherin $m R N A$ expression. The 3 mRNAs were all upregulated, without a statistically significant difference ( $\mathrm{p}>0.05)$ (Fig. 2D).

\section{Discussion}

\section{Expression profile of integrin $m R N A s$}

Expression profile of leukocyte-related integrin mRNAs. Among the 13 mRNAs, $5(38.46 \%)$ were upregulated with a statistically significant difference $(\mathrm{p}<0.05)$. In these 5 mRNAs, the ITGAL, ITGAM and ITGAX transcripts (subunit $\alpha \mathrm{L}, \alpha \mathrm{M}$ and $\alpha \mathrm{X}$ ) bind to subunit $\beta 2$ to generate $\beta 2$ integrins, and are expressed on leukocytes specifically; the ITGA5 and ITGB1 transcripts are subunits of $\alpha 5$ and $\beta 1$. $\beta 1$ binds to $\alpha 1-\alpha 11$ and $\alpha \mathrm{V}$ to generate $\beta 1$ integrins (13).

The $3 \alpha$ subunits of $\beta 2$ integrins were all significantly upregulated with a statistically significant difference, indicating that connections between $\beta 2$ integrins and their ligands were enhanced during the interaction between leukocytes and vascular endothelial cells, a fact that suggests that the adhesion of $\beta 2$ integrins was enhanced. In the $\beta 1$ integrins, only $\alpha 5$ and $\beta 1$ were significantly upregulated with a statistically significant difference. $\alpha 5 \beta 1$ was also expressed in platelets, 
A

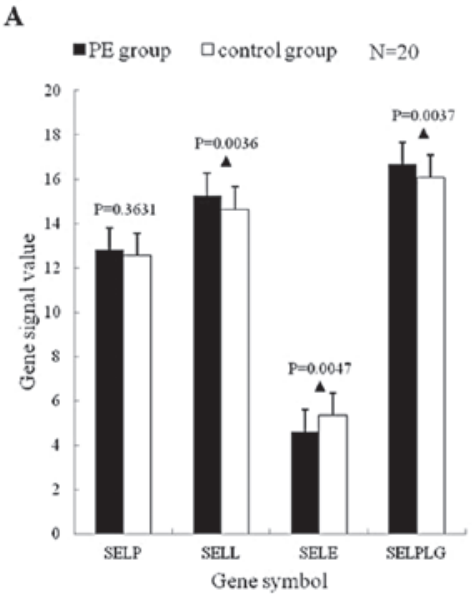

D

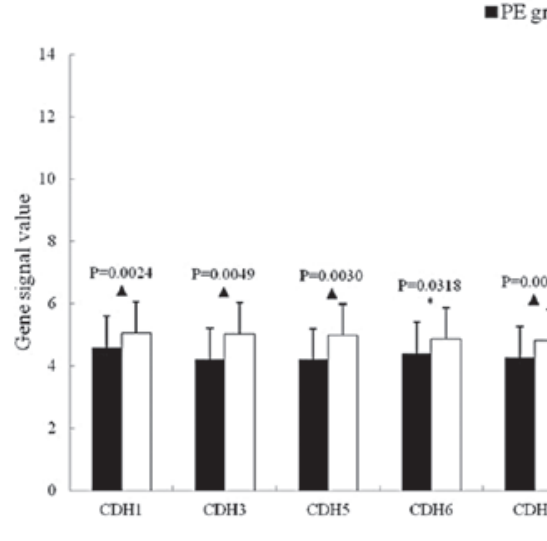

B

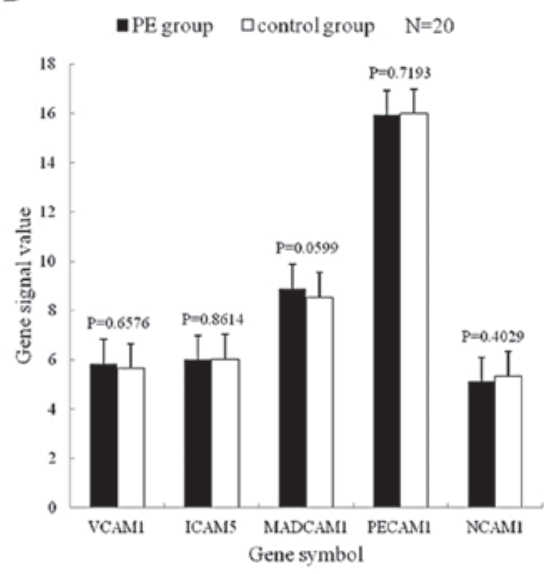

C

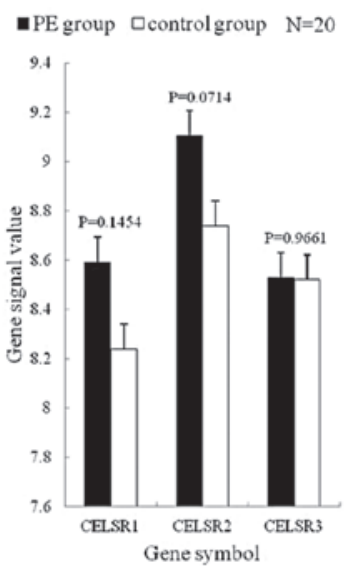

PE group $\quad \mathrm{N}=20$

Figure 2. Expression of selectin, immunoglobulin superfamily, flamingo cadherin and classic cadherin mRNAs. (A) Selectin mRNA expression; (B) immunoglobulin superfamily mRNA expression; (C) flamingo cadherin mRNA expression; (D) classic cadherin mRNA expression. ${ }^{*} \mathrm{p}<0.05$; ${ }^{\wedge} \mathrm{p}<0.01$.

indicating that the upregulation of the subunits $\alpha 5$ and $\beta 1$ occurred in leukocytes and platelets at the same time.

Thus, we considered that the signal transduction of $\beta 1$ integrins was enhanced and leukocytes actively reinforced adhesion to the vascular endothelial cells in PE patients. The $\alpha \mathrm{M}$ and $\alpha \mathrm{X}$ mRNAs were significantly upregulated. $\alpha \mathrm{M} \beta 2$ was expressed in monocytes, macrophages and neutrophils, while $\alpha \mathrm{X} \beta 2$ was expressed in monocytes, NK cells, neutrophils and some lymphocytes. Caimi et al reported that neutrophils were activated abnormally in VTE $(14,15)$. The genomics results were consistent with the cytology results.

Expression profile of platelet-related integrin mRNAs. There are 5 integrins expressed in platelets: $\alpha \mathrm{II} \beta 3$ (GPIIb/IIIa), $\alpha \mathrm{V} \beta 3, \alpha 2 \beta 1, \alpha 5 \beta 1$ and $\alpha 6 \beta 1$. In our research, the 2 subunits of GPIIb/IIIa were both significantly upregulated. Most GPIIb/IIIa copies are present on resting platelets, while another small part is hidden in platelet $\alpha$-granules. The GPIIb/IIIa copies on $\alpha$-granules become externalized on platelet secretion to increase their surface expression by 25 to $50 \%$, and expose their binding sites for fibrinogens to become activated (16).

Expression profile of other integrin mRNAs. In these 11 mRNAs, the ITGA11 and ITGA7 transcripts $(\alpha 11$ and $\alpha 7)$ bind to subunit $\beta 1$ to generate $\alpha 11 \beta 1$ and $\alpha 7 \beta 1$; the ITGB5 ( $\beta 5$ ) transcript binds to subunit $\alpha \mathrm{V}$ to generate $\alpha \mathrm{V} \beta 5$. The ligands of $\alpha 11 \beta 1, \alpha 7 \beta 1$ and $\alpha \mathrm{V} \beta 5$ are collagen, laminin and fibronectin, respectively (17), indicating that the signal transduction and the binding of integrins to collagen, laminin and fibronectin are enhanced in PE patients. In the 3 mRNA transcripts which were significantly downregulated, the subunit $\alpha 8$ binds to $\beta 1$ to generate $\alpha 8 \beta 1 ; \beta 6$ and $\beta 8$ bind to $\alpha \mathrm{V}$ to generate $\alpha \mathrm{V} \beta 6$ and $\alpha \mathrm{V} \beta 8$. Major ligands of $\alpha 8 \beta 1, \alpha \mathrm{V} \beta 6$ and $\alpha \mathrm{V} \beta 8$ are all fibronectin.

In the PE group, most upregulated mRNAs of the leukocyte-related integrin transcribed into $\beta 1$ and $\beta 2$ integrins; $60 \%$ of platelet-related integrin mRNAs were upregulated which transcribed into $\beta 1$ and $\beta 3$ integrins, indicating that integrins expressed in leukocytes and platelets play an important role in the PE process, while the upregulation of $\beta 1$ integrin mRNAs was related to both leukocytes and platelets. Most transcripts of other integrin mRNAs which were abnormally expressed bound to fibronectin, indicating that fibronectin plays a role in the onset of VTE.

Expression profile of selectin $m R N A s$. P-selectin is stored in the a-granules of platelets and the Weibel-Palade bodies of endothelial cells (18); E-selectin is expressed on the surface of activated leukocytes specifically (19); L-selectin is expressed on the surface of most leukocytes (20); P-selectin glycoprotein ligand (PSGL-1) is expressed mainly on the surface of leukocytes and platelets (21). PSGL-1 is a receptor of P-selectin with 
A

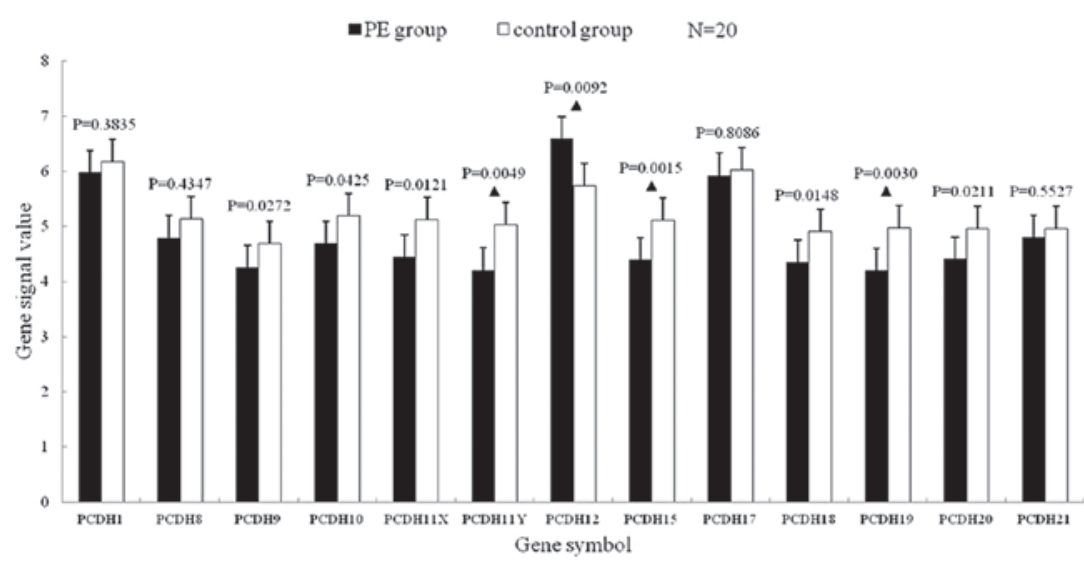

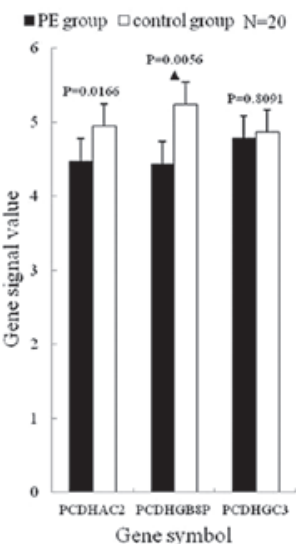

C

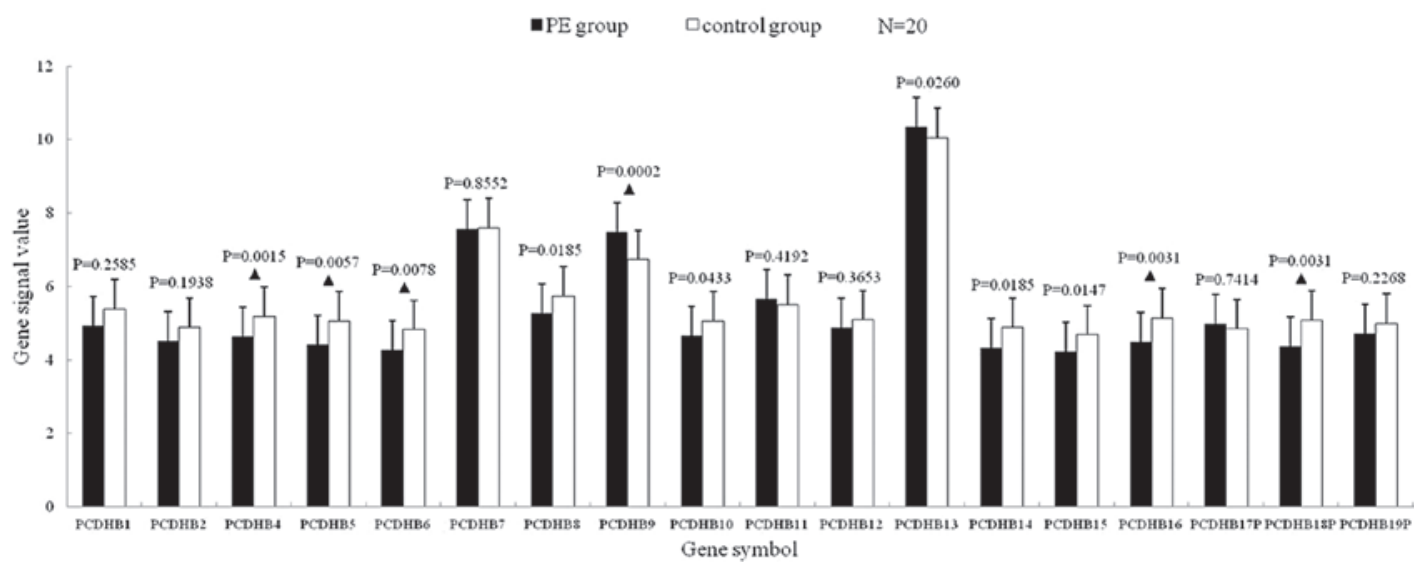

Figure 3. Expression of protocadherin mRNAs. (A) Non-clustered protocadherin mRNA expression; (B) clustered protocadherin $\alpha$ and $\gamma$ mRA expression; (C) clustered protocadherin $\beta$ mRNA expression. ${ }^{\mathrm{p}} \mathrm{p}<0.05 ;{ }^{\wedge} \mathrm{p}<0.01$.

high affinity that can bind both to L-selectin and E-selectin to mediate interactions among leukocytes, platelets and endothelial cells. In our research, L-selectin and PSGL-1 mRNAs were significantly upregulated, indicating that leukocytes were activated in PE patients; E-selectin mRNA was significantly downregulated, indicating that the activation of endothelial cells was obviously weakened, while no statistically significant difference in P-selectin between the PE and the control group was observed.

Expression profile of immunoglobulin superfamily mRNAs. The expression trends of VCAM-1 mRNA and PECAM-1 mRNA in PE patients were similar to those in artery thrombosis (22); no statistically significant difference in the expression of MadCAM-1, ICAM-1, NCAM-1 between the $\mathrm{PE}$ and the control group was observed, indicating that the immunoglobulin superfamily did not play an important role in PE.

\section{Expression profile of cadherin superfamily $m R N A s$}

Expression profile of classic cadherin mRNAs. Among the 13 mRNAs, 7 (53.84\%) mRNAs were downregulated statistically, of which $4(30.77 \%)$ significantly. Vascular endothelial cell cadherin (VE-cadherin) expressed on the surface of endothelial cells is the major endothelial adhesion molecule of the adherens junction, and negatively regulates the transendothelial migration of leukocytes (23). During inflammation, leukocytes weaken the negative regulatory effect of VE-cadherin by VE-cadherin phosphorylation (24), as well as the binding of VE-cadherin to endothial cells $(25,26)$ and cleave VE-cadherins $(27)$ to promote their transendothelial migration, enhance leukocyte infiltration and cause inflammation. In this research, VE-cadherin mRNA expression was significantly downregulated, indicating that the adherens junction between vascular endothelial cells was obviously weakened and that the permeability of the endothelium was increased in PE patients.

Expression profile of protocadherin $m R N A s$. A total of $19(55.88 \%)$ mRNAs were significantly downregulated among the 34 protocadherin mRNAs $(\mathrm{p}<0.05)$, of which 9 (26.47) more significantly $(\mathrm{p}<0.01)$, indicating that protocadherins were downregulated as a whole in VTE. The result was similar to classic cadherins. Similar to VE-cadhrein, VE-cadherin 2 was also expressed on the adherens junction among endothelial cells; however, in contrast to VE-cadherin, its mRNA expression was upregulated in PE patients.

Expression profile of flamingo cadherin mRNAs. Flamingo cadherins are a type of 7-pass transmembrane protein (28). In this research, the 3 mRNAs of this sub-family were all upregulated in PE patients, but with no statistically significant difference. 
During the process of PE, leukocytes were significantly activated with enhanced adhesion, the adhesion of platelets was enhanced and the activation of vascular endothelial cells and the adherens junction between endothelial cells were weakened, with the endothelium becoming more permeable. During the process of PE, leukocytes increased their ability to adhere and were assisted by platelet adhesion. The vascular endothelial cell adhesion, however, may be passive. The obvious weakness of the adherens junction among endothelial cells provided the conditions for the migration of inflammatory cells.

\section{Acknowledgements}

The present study was supported by the National Natural Science Foundation of China (No. 30570809) and the Key Foundation of Science and Technology Commission of the Shanghai Municipality (No. 05JC14038)

\section{References}

1. Spencer FA, Gore JM, Lessard D, Douketis JD, Emery C and Goldberg RJ: Patient outcomes after deep vein thrombosis and pulmonary embolism: the Worcester Venous Thromboembolism Study. Arch Intern Med 168: 425-430, 2008.

2. Virchow RLK: Thrombosis and emboli. Science History Publications, Massachusetts, MA, 1998.

3. Geerts WH, Pineo GF, Heit JA, Bergqvist D, Lassen MR, Colwell CW and Ray JG: Prevention of venous thromboembolism: the Seventh ACCP Conference on Antithrombotic and Thrombolytic Therapy. Chest 126 (Suppl 3): 338S-400S, 2004.

4. Geerts WH, Bergqvist D, Pineo GF, Heit JA, Samama CM Lassen MR and Colwell CW; American College of Chest Physicians.: Prevention of venous thromboembolism: American College of Chest Physicians Evidence-Based Clinical Practice Guidelines (8th Edition). Chest 133 (Suppl 6): 381S-453S, 2008.

5. Shackford SR, Rogers FB, Terrien CM, Bouchard P, Ratliff J and Zubis R: A 10-year analysis of venous thromboembolism on the surgical service: the effect of practice guidelines for prophylaxis. Surgery 144: 3-11, 2008.

6. Smeeth L, Cook C, Thomas S, Hall AJ, Hubbard R and Vallance P: Risk of deep vein thrombosis and pulmonary embolism after acute infection in a community setting. Lancet 367: 1075-1079, 2006.

7. Xiang-Hua Y, Le-Min W, Ai-Bin L, Zhu G, Riquan L, Xu-You Z, Wei-Wei R and Ye-Nan W: Severe acute respiratory syndrome and venous thromboembolism in multiple organs. Am J Respir Crit Care Med 182: 436-437, 2010.

8. Wang L, Gong Z, Liang A, Xie Y, Liu SL, Yu Z, Wang L and Wang Y: Compromised T-cell immunity and virus-like structure in a patient with pulmonary hypertension. Am J Respir Crit Care Med 182: 434-435, 2010.

9. Haoming S, Lemin W, Zhu G, Aibin L, Yuan X, Wei L, Jinfa J, Wenjun $X$ and Yuqin S: T cell-mediated immune deficiency or compromise in patients with CTEPH. Am J Respir Crit Care Med 183: 417-418, 2011.

10. Wang L, Gong Z, Jiang J, Xu W, Duan Q, Liu J and Qin C: Confusion of wide thrombolytic time window for acute pulmonary embolism: mass spectrographic analysis for thrombus proteins. Am J Respir Crit Care Med 184: 145-146, 2011.
11. Prescott SM, Weyrich AS and Zimmerman GA: Classification of venous thromboembolism (VTE). The clot is hot: inflammation, myeloid leukocytes, and venous thromboembolism. J Thromb Haemost 3: 2571-2573, 2005.

12. Henke PK and Wakefield T: Thrombus resolution and vein wall injury: dependence on chemokines and leukocytes. Thromb Res 123 (Suppl 4): S72-S78, 2009.

13. Takada Y, Ye X and Simon S: The integrins. Genome Biol 8: 215 , 2007.

14. Caimi G, Tozzi Ciancarelli MG, Ferrara F, Montana M, Calandrino V, Canino B and Lo Presti R: Deep venous thrombosis: behaviour of the polymorphonuclear leukocyte integrin pattern at baseline and after in vitro activation. Clin Hemorheol Microcirc 33: 11-17, 2005.

15. Caimi G, Canino B, Ferrara F, Montana M and Lo Presti R: Polymorphonuclear leukocyte integrins in deep venous thrombosis. Clin Appl Thromb Hemost 11: 95-97, 2005.

16. Quinn MJ, Byzova TV, Qin J, Topol EJ and Plow EF: Integrin alphaIIbbeta3 and its antagonism. Arterioscler Thromb Vasc Biol 23: 945-952, 2003.

17. Faralli JA, Schwinn MK, Gonzalez JM Jr, Filla MS and Peters DM: Functional properties of fibronectin in the trabecular meshwork. Exp Eye Res 88: 689-693, 2009.

18. Wagner DD: The Weibel-Palade body: the storage granule for von Willebrand factor and P-selectin. Thromb Haemost 70: 105-110, 1993.

19. Bevilacqua MP, Stengelin S, Gimbrone MA Jr and Seed B: Endothelial leukocyte adhesion molecule 1: An inducible receptor for neutrophils related to complement regulatory proteins and lectins. Science 243: 1160-1165, 1989.

20. Kansas GS: Selectins and their ligands: Current concepts and controversies. Blood 88: 3259-3287, 1996.

21. Wakefield TW, Myers DD and Henke PK: Mechanisms of venous thrombosis and resolution. Arterioscler Thromb Vasc Biol 28: 387-391, 2008.

22. Gong $\mathrm{N}$ and Chatterjee S: Platelet endothelial cell adhesion molecule in cell signaling and thrombosis. Mol Cell Biochem 253: 151-158, 2003.

23. Muller WA: Mechanisms of transendothelial migration of leukocytes. Circ Res 105: 223-230, 2009.

24. Nottebaum AF, Cagna G, Winderlich M, Gamp AC, Linnepe R, Polaschegg C, Filippova K, Lyck R, Engelhardt B, Kamenyeva O, et al: VE-PTP maintains the endothelial barrier via plakoglobin and becomes dissociated from VE-cadherin by leukocytes and by VEGF. J Exp Med 205: 2929-2945, 2008.

25. Boggon TJ, Murray J, Chappuis-Flament S, Wong E, Gumbiner BM and Shapiro L: C-cadherin ectodomain structure and implications for cell adhesion mechanisms. Science 296: 1308-1313, 2002

26. Hermant B, Bibert S, Concord E, Dublet B, Weidenhaupt M Vernet T, Gulino-Debrac D: Identification of proteases involved in the proteolysis of vascular endothelium cadherin during neutrophil transmigration. J Biol Chem 278: 14002-14012, 2003.

27. Dejana E, Orsenigo F and Lampugnani MG: The role of adherens junctions and VE-cadherin in the control of vascular permeability. J Cell Sci 121: 2115-2122, 2008.

28. Shima Y, Kengaku M, Hirano T, Takeichi M and Uemura T: Regulation of dendritic maintenance and growth by a mammalian 7-pass transmembrane cadherin. Dev Cell 7: 205-216, 2004. 